\title{
Potential of combating transmission of COVID-19 using novel self-cleaning superhydrophobic surfaces: part II-thermal, chemical, and mechanical durability
}

\author{
Assem Elzaabalawy $\mathbb{D} \cdot$ S. A. Meguid
}

Received: 13 July 2020/Accepted: 27 July 2020/Published online: 5 August 2020

(C) Springer Nature B.V. 2020

\begin{abstract}
In part I, we identified encapsulation, contamination suppression, and virus elimination as our three governing strategies for developing surfaces to combat the transmission and spread of COVID-19. We showed that our recent superhydrophobic nanocomposites has the potential of encapsulating and suppressing the virus so as to limit its transmission and spread. In this study, we examine the durability of the newly developed surfaces when subjected to elevated temperature, chemical attack and mechanical damage in the form of abrasion and compressive load. Extensive tests were conducted to reveal the effect of these parameters on the surface performance. Three aspects of the work were accordingly examined. The first was concerned with controlled thermal stability tests in which the surfaces were subjected to elevated temperatures approaching $350{ }^{\circ} \mathrm{C}$ for silicone-based nanocomposites and $150{ }^{\circ} \mathrm{C}$ for epoxy-based nanocomposites. The second was concerned with subjecting the surfaces to alkaline and acidic solutions with $\mathrm{pH}$ concentrations ranging between 1 and 13 . Finally, the third involved surface damage by abrasion tests. Our results show clearly that the newly developed superhydrophobic surfaces are capable of resisting the adverse effects of thermal and chemical attacks
\end{abstract}

A. Elzaabalawy $\cdot$ S. A. Meguid ( $\square)$

Mechanics and Aerospace Design Lab, University of

Toronto, 5 King's College Rd, Toronto,

ON M5S 3G8, Canada

e-mail: meguid@mie.utoronto.ca as well as mechanical abrasion owing to the excellent structural stability and mechanical properties of the constituents of the nanocomposite. Moreover, our superhydrophobic monolith demonstrated exceptional regenerative capabilities even after being subjected to damaging compressive stresses of up to $10 \mathrm{MPa}$.

Keywords COVID-19 - Combat .

Superhydrophobic · Thermal · Chemical . Mechanical · Durability

\section{Introduction}

COVID-19 can be transmitted through airborne respiratory droplets, ejected as a result of coughing or sneezing through human contact with contaminated surfaces (Yang and Wang 2020; Gralinski and Menachery 2020). Recent studies by the National Institute of Allergy and Infectious Diseases (USA) have shown that the virus can infect fomites made of metals, polymers and recycled paper for days (Van Doremalen et al. 2020).

Superhydrophobic surfaces have recently gained popularity in the biomedical sector due to their ability to possess blood repellency characteristics and reduce bacterial/viral adhesion to surfaces and their antifouling properties (Falde et al. 2016; Shin et al. 2016; Jaggessar et al. 2017). Earlier studies have shown that 
personal protective equipment (PPE) that have high contact angles (CA) and low sliding angles (SA) can significantly reduce a virus's carryover potential and provide antimicrobial properties (Katoh et al. 2019; Tomšič et al. 2008; Yeerken et al. 2019).

In Part I, we demonstrated the potential in creating superhydrophobic coatings and monoliths that are capable to combating the transmission and spread of COVID-19. However, one of the main challenges that restricts the widespread utilization of superhydrophobic coatings in real applications is their durability (Ellinas et al. 2017; Jeevahan et al. 2018). Specifically, thermal and chemical stability as well as mechanical strength. Elevated temperatures can lead to the thermal degradation of hydrocarbon coatings (Cha et al. 2013) or thermal desorption of the hydrophobic material (Saleema and Farzaneh 2008), which result in the loss of surface superhydrophobicity. Additionally, some polymeric superhydrophobic surfaces cannot withstand elevated temperatures and are not recommended to operate above their glass transition temperature (Ellinas et al. 2017).

Similarly, chemical attack due to exposure to acidic or alkaline solutions can result in severe deterioration of the superhydrophobicity of the treated surfaces (BaratiDarband et al. 2020). For instance, highly alkaline or acidic solutions can result in dissolving the hydrophobic functional groups (Zhao et al. 2017; Ishizaki et al. 2010) and cause chemical desorption of the hydrophobic material (Cui et al. 2015).

Mechanical wear is considered one of the main performance degradation mechanisms of superhydrophobic surfaces (Milionis et al. 2016). Indeed, numerous studies and tests have been conducted to evaluate the influence of abrasion tests on the mechanical durability of these surfaces (Ellinas et al. 2017; Mortazavi and Khonsari 2017). Superhydrophobic surfaces generally suffer from weak mechanical durability due to the fragility of their nano/microhierarchical surface structure (Mortazavi and Khonsari 2017; Zhang et al. 2013). Additionally, polymeric materials involved in fabricating superhydrophobic surfaces usually suffer from poor mechanical stability and robustness (Ellinas et al. 2017). In our previous work (Elzaabalawy et al. 2019), we demonstrated that high external compressive loads can also lead to permanent plastic deformation of the surface hierarchical structure and the embedment of the hydrophobic nanoparticles, which ultimately cause a complete loss of superhydrophobicity.

\section{Hypothesis}

COVID-19 spreads mainly through droplet transmission or via contacting fomites. We believe that surfaces coated with or created from a superhydrophobic nanocomposite has the potential of combating virus transmission and spread through a 3-step strategy discussed in Part I. The superhydrophobic nanocomposites acquire their protection characteristics through chemical modification as well as a hierarchical nano/ microstructure of the modified surface. However, typical surfaces are subjected to elevated temperatures, chemical attacks and mechanical damage during service. As a result, the surface repellency characteristics may be altered and the effectiveness and the potential of the surface to encapsulate and suppress the virus might be lost. For nanocomposite superhydrophobic surfaces, utilizing different polymeric materials can be used to vary the flexibility and durability of the developed surfaces. For instance, silicone polymers can be used when flexibility is sought, and epoxy can be employed to meet the requirements of harsh mechanical environments. The durability of the nanocomposite can also be enhanced and tailored by selecting constituent materials that possess high thermal and chemical stability. Moreover, superhydrophobic monoliths with regenerative capabilities can be employed in severe service conditions to accommodate mechanical damage. Since these monoliths acquire water repellency features throughout the entire bulk of the material, surfaces can be regenerated by removing the damaged layer and exposing a new superhydrophobic layer. In this case, superhydrophobicity can be maintained as long as the monolith's thickness can accommodate a layer removal.

\section{Objectives}

The aim of Part II is to assess the ability of the superhydrophobic coating and monoliths discussed in Part I to resist thermal degradation, chemical attacks, and mechanical damage. Three aspects of the work were accordingly examined. The first was concerned 
with thermal stability tests in which the superhydrophobic surfaces were subjected to elevated temperatures approaching $350{ }^{\circ} \mathrm{C}$ for silicone-based nanocomposites and $150{ }^{\circ} \mathrm{C}$ for epoxy-based nanocomposites. The second was concerned with subjecting the surfaces to alkaline and acidic environments with $\mathrm{PH}$ values ranging between 1 and 13 . Finally, the mechanical durability of the surface of epoxy-based nanocomposite coating was assessed using abrasion tests, while the regenerative capability of the silicone-based nanocomposite monoliths was evaluated using compressive loading. Given below is a summary of the approach adopted and the outcomes of our research.

\section{Durability and protection of superhydrophobic surfaces}

In this research program, superhydrophobic coatings and monoliths were developed using epoxy and silicone nanocomposites. We demonstrated the ability of these coatings to modify the surface characteristics of different substrate materials, as shown in Fig. 1. We have also succeeded in fabricating a bulk that is entirely superhydrophobic. In order to assess the thermal, chemical, and mechanical stability of these surfaces, multiple tests were conducted.

Since superhydrophobic surfaces are characterized by $\mathrm{CA}$ above $150^{\circ}$ and SA below $10^{\circ}$, the superhydrophobicity in these tests was assessed by measuring the contact and sliding angles. Contact angles were measured using an OCA 15EC contact angle analyzer (DataPhysics Instruments, Fig. 2), while sliding angles were measured using an in-house apparatus with controlled surface tilting until droplets roll off is achieved.

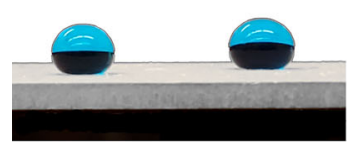

(a)

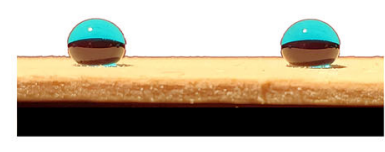

(b)

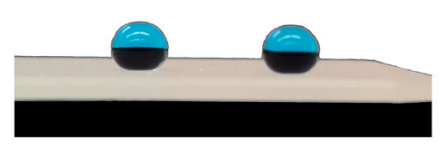

(c)

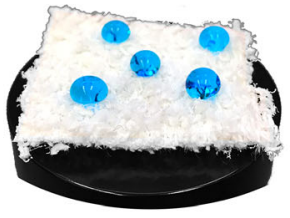

(d)

Fig. 1 Static water droplets resting on different substrate materials covered with the epoxy-based nanocomposite superhydrophobic coating: a metal b wood, c glass, and $\mathbf{d}$ fabric 


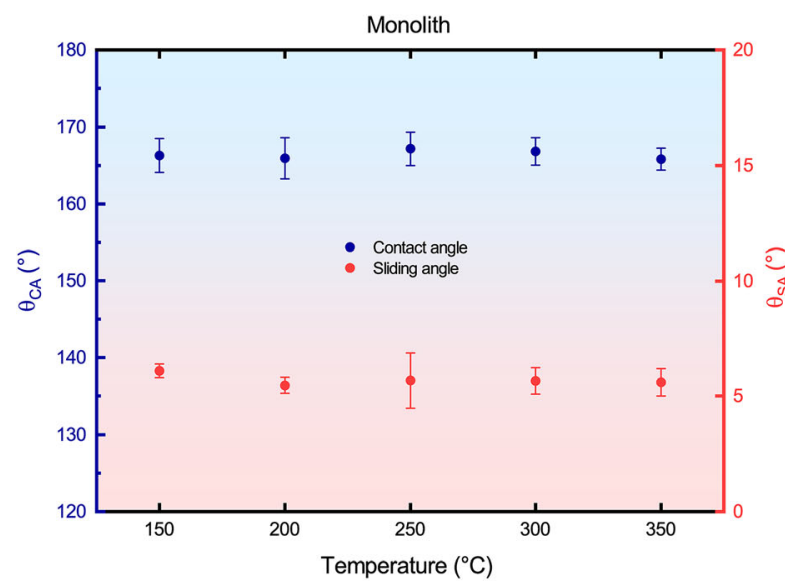

(a)

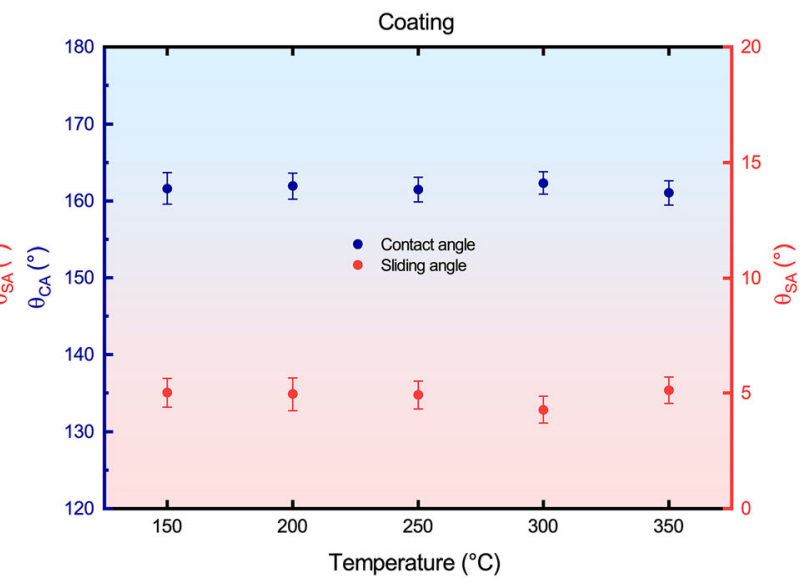

(b)

Fig. 4 Thermal durability test results for silicone-based a coating and $\mathbf{b}$ monolith

superhydrophobic surface to ensure that water repellency is maintained after being subjected to surges of elevated temperatures. Unlike organic polymers, silicone is characterized by its higher thermal resistance due to the high stability of its siloxane backbone.

In order to examine the thermal durability, coated samples and monoliths were placed in an oven for $3 \mathrm{~h}$. Tests were conducted for different temperatures and the maximum temperature was selected based on the polymer's auto-ignition temperature. Upon completing the test, samples were left to cool down, and then the contact and sliding angles were re-evaluated to assess their superhydrophobicity characteristics.

For epoxy-based nanocomposites, thermal durability tests were conducted for temperatures between 75 and $150{ }^{\circ} \mathrm{C}$ in $25^{\circ} \mathrm{C}$ increments. As depicted in Fig. 3, no variation was observed to the values of $\mathrm{CA}$ $\left(\sim 165^{\circ}\right)$ or SA $\left(\sim 3^{\circ}\right)$ for all of the tested temperatures. These results verify that the coated samples maintained their superhydrophobicity and the siloxane-modification process (Part I) succeeded in enhancing the water repellency of epoxy without compromising its durability.

Silicone-based nanocomposites were tested for temperatures between $150{ }^{\circ} \mathrm{C}$ and $350{ }^{\circ} \mathrm{C}$ in $50{ }^{\circ} \mathrm{C}$ increments. As shown in Fig. 4, the coated samples and monoliths maintained high CA $\left(\sim 167^{\circ}\right.$ for monoliths and $\sim 163^{\circ}$ for coatings) and low SA $\left(6^{\circ}\right.$ for monoliths and $5^{\circ}$ for coatings) throughout the entire temperature range. These results further demonstrate the capability of the strong siloxane backbone within the polymer chemical structure to withstand elevated temperatures and maintain the superhydrophobicity of the nanocomposite.

\section{Chemical stability tests}

Chemical attacks or short-term immersion in corrosive solutions can result in severe degradation of the surface characteristics. To assess the chemical stability of the developed superhydrophobic nanocomposites, samples were immersed in acidic and alkaline solutions for $3 \mathrm{~h}$ at room temperature in a fume hood, as depicted in Fig. 5. Solutions were prepared using sulphuric acid or sodium hydroxide, which were diluted using distilled water to achieve $\mathrm{pH}$ values between 1 and 13. Following the immersion, water repellency of the samples was again evaluated by measuring the contact and sliding angles.

Following the immersion tests, samples were observed to check their reflective appearance in the 


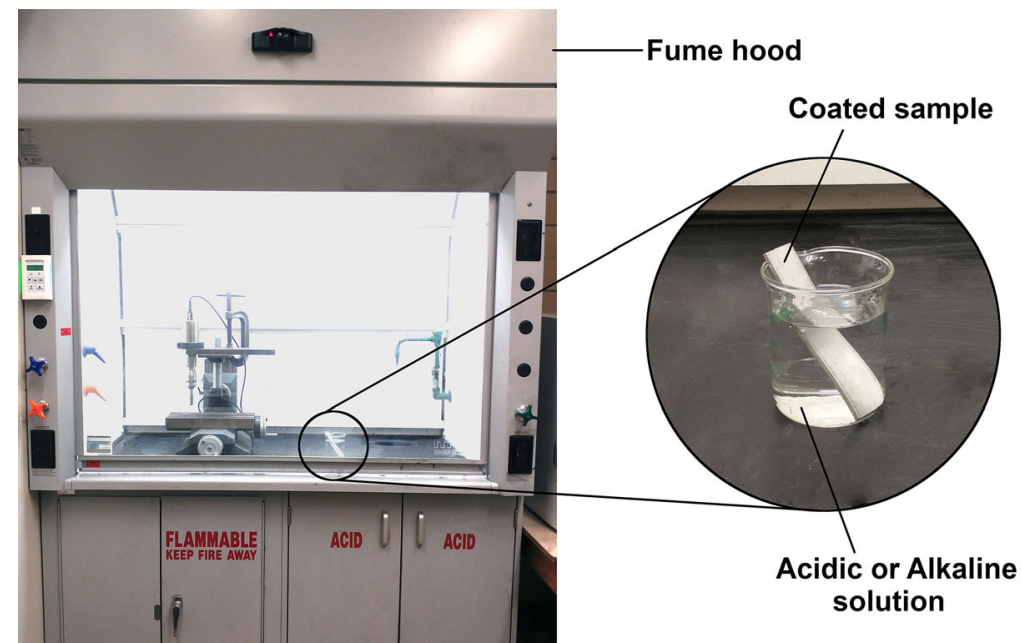

Fig. 5 Chemical stability tests at room temperature

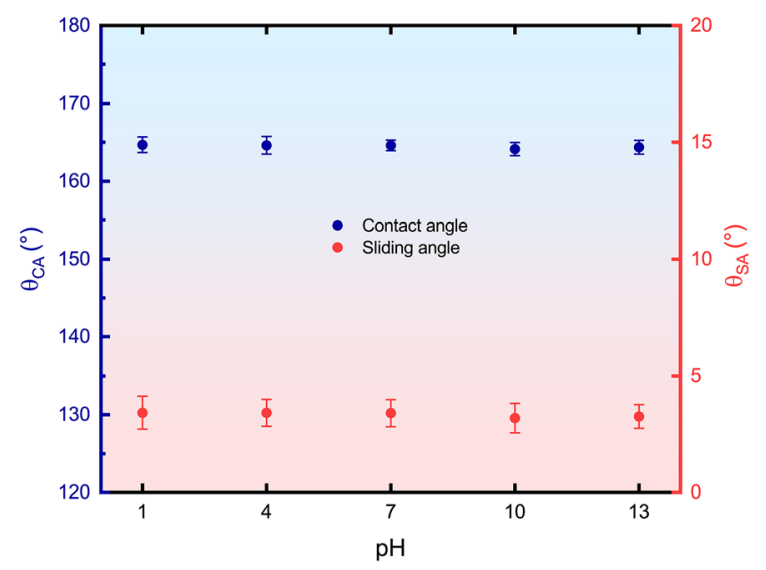

solution, which indicates a stable Cassie-Baxter wetting state, and to be completely dry upon removal. Furthermore, following the immersion, the CA and SA were measured for the epoxy-based and silicone-based nanocomposites, as depicted in Figs. 6 and 7, respectively. The measurements revealed that the nanocomposites are capable of maintaining their excellent superhydrophobicity for the entire range of $\mathrm{pH}$ values tested. Accordingly, these nanocomposites can operate and effectively maintain their performance in harsh service conditions that are subjected to acidic or alkaline chemical attacks.

Fig. 6 Chemical durability test results for epoxy-based coatings

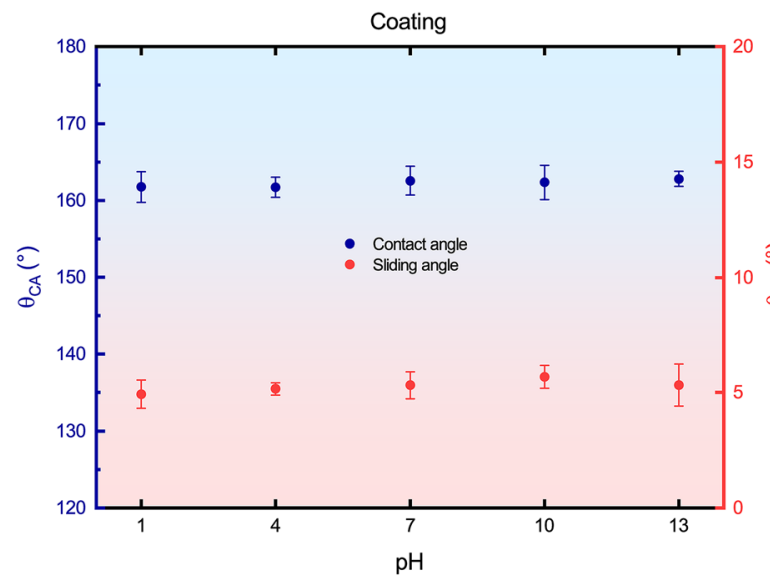

(a)

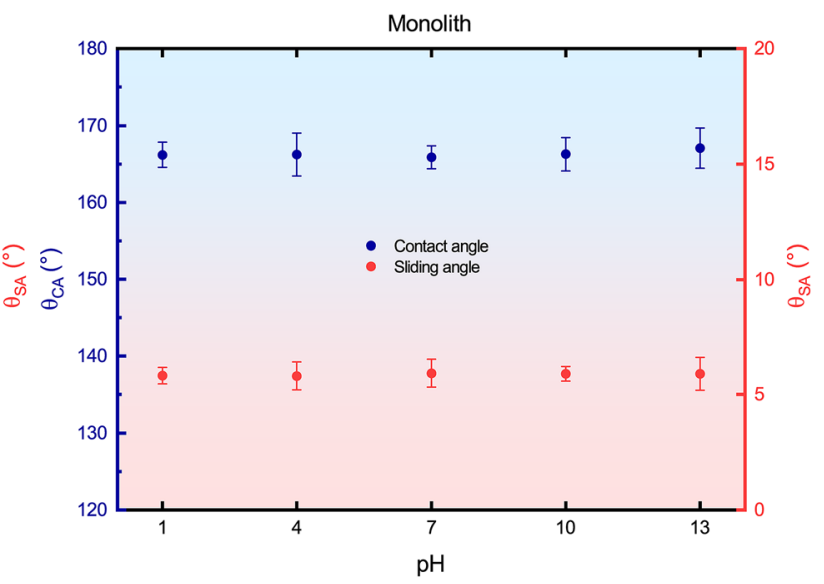

(b)

Fig. 7 Chemical durability test results for silicone-based a coating and $\mathbf{b}$ monolith 


\section{Mechanical strength and durability tests}

Abrasion is considered the most widely test used to evaluate the mechanical properties of superhydrophobic coatings (Milionis et al. 2016). Since siliconebased coatings are sought to provide flexibility rather than mechanical strength, abrasion tests were only conducted to evaluate the strength and durability of epoxy-based nanocomposite coatings. As schematically shown in Fig. 8, the samples were placed face down on silicon carbide coarse sandpaper (320 grit) under a calibrated weight pressure of $\sim 1 \mathrm{kPa}$. Abrasion cycles (10 cm of linear displacement) were performed in alternating directions. Following each interval of cycles, debris were removed, and the contact and sliding angles were measured.

The contact and sliding angles measurements following abrasion cycle intervals are demonstrated in Fig. 9. After 120 abrasion cycles, the coating was only able to maintain its high contact angle $\left(>150^{\circ}\right)$, while the sliding angle increased above $10^{\circ}$. In order to examine the degradation caused to the nano/microstructure of the surface, SEM images were obtained,

Figure 10 the results indicate that excessive abrasion cycles induce damage to the surface's hierarchical structure (Fig. 9b) compared to the original

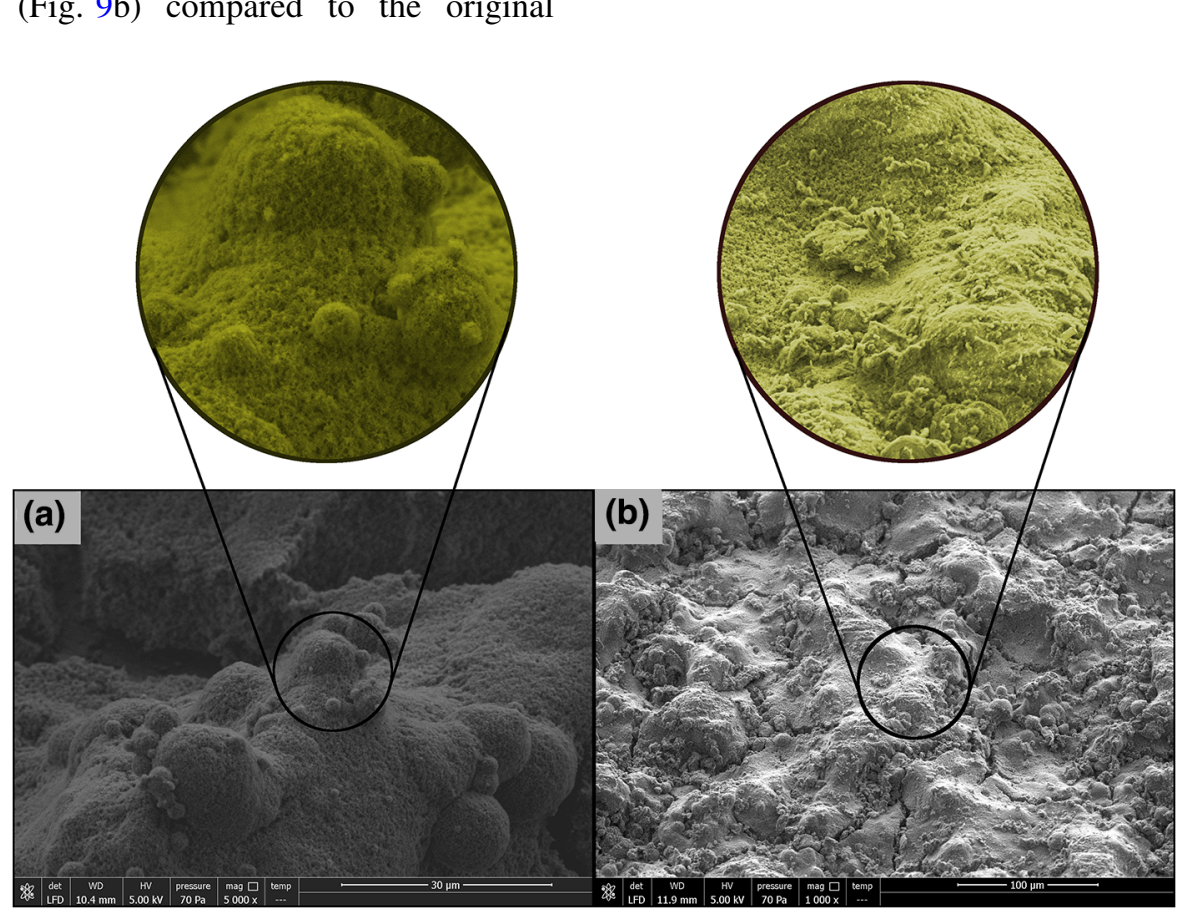

Fig. 10 SEM images of superhydrophobic coating a prior to abrasion and $\mathbf{b}$ after 120 abrasion cycles
Calibrated applied weight

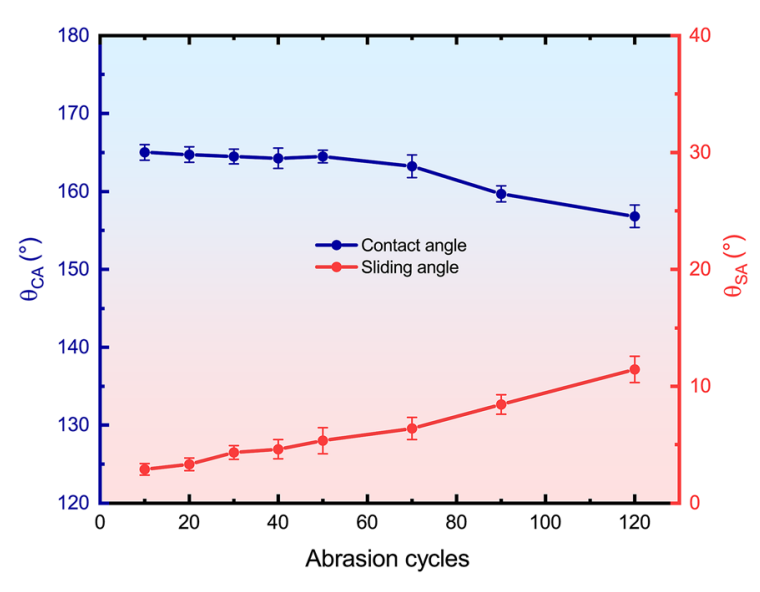

Fig. 9 Abrasion test results for epoxy-based coatings
Fig. 8 A schematic of abrasion test conducted

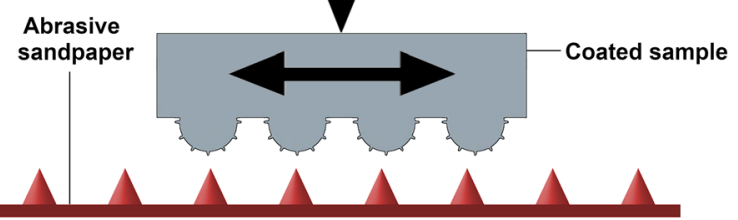


superhydrophobic untouched coating (Fig. 9a). However, the nanocomposite coating was able to superbly sustain its superhydrophobicity after 90 abrasion cycles, which is attributed to the excellent adhesion strength and mechanical properties of epoxy polymers.

\section{Regenerative capability of monolith}

Although the epoxy-based nanocomposite coating demonstrated excellent mechanical durability characteristics, service conditions can sometimes be harsher than a coating can handle. In those situations, a silicone-based nanocomposite monolith that is entirely superhydrophobic is an excellent replacement. Under harsh mechanical environment, the superhydrophobic surface's hierarchical structure would be damaged. To obviate this damage, the surface can overcome that damage by the regeneration of a fresh superhydrophobic layer under controlled abrasion. In order to demonstrate these regenerative capabilities, monoliths were exposed to compressive stresses ranging between 1 and $10 \mathrm{MPa}$ with the aid of a uniaxial electrohydraulic servo-controlled testing machine (Instron 5965). Samples with a square crosssection $(20 \mathrm{~mm} \times 20 \mathrm{~mm})$ were placed between two flat metallic plates, and then the loading was applied at a displacement rate of $1 \mathrm{~mm} / \mathrm{min}$ until the desired load

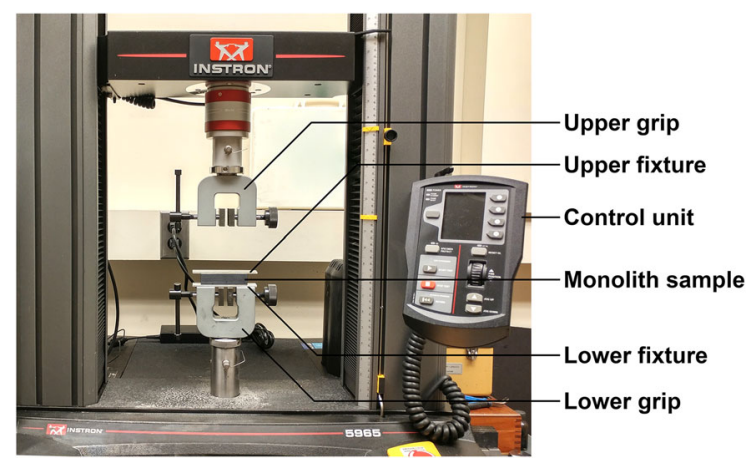

Fig. 11 Mechanical testing of superhydrophobic monoliths was obtained. The compressive load was then maintained for $5 \mathrm{~min}$ before the samples were unloaded. Subsequently, CA and SA were measured after unloading and after samples were regenerated. The test set up is depicted in Fig. 11.

The compression test results (Fig. 12) revealed that the monolith can withstand stresses up to $1 \mathrm{MPa}$ without affecting its superhydrophobicity, in which $\mathrm{CA}$ and SA are maintained at $\sim 167^{\circ}$ and $6^{\circ}$ respectively. For increased compressive stresses (3 and $5 \mathrm{MPa}$ ), gradual decrease in the $\mathrm{CA}$ and increase in SA were noticed. At a compressive stress of $10 \mathrm{MPa}, \mathrm{CA}$ reached $\sim 149^{\circ}$ and droplets were pinned to the surface and did not roll off even when applying a finite inclination angle. As shown in Fig. 13, SEM images indicated that this deterioration was caused by the plastic deformation in the surface's hierarchical structure as a result of the high compressive stresses. Remarkably, the surface's superhydrophobicity was fully restored upon the removal of the damaged layer through abrasion, and the respective CA and SA were fully recovered to $\sim 167^{\circ}$ and $6^{\circ}$, as depicted in Fig. 12. As observed, the regeneration process was successful for all values of destructive stresses (3-10 MPa).

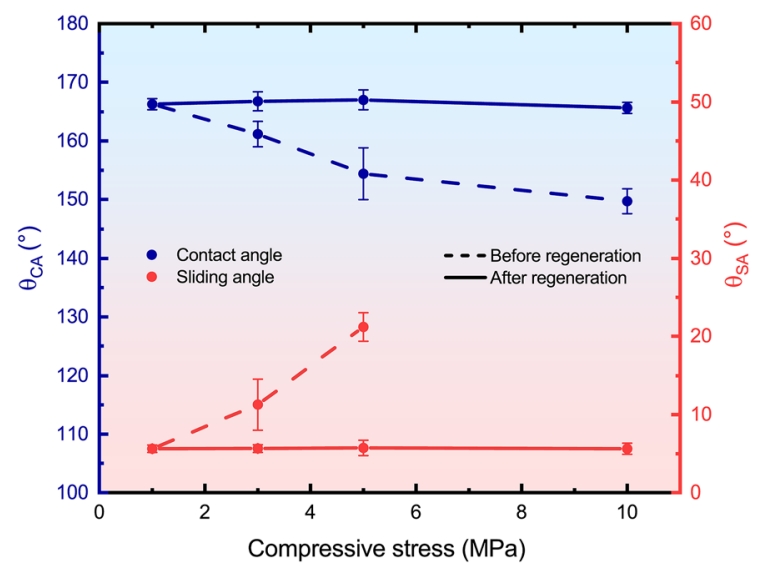

Fig. 12 Compression and regeneration test results for siliconebased monoliths 


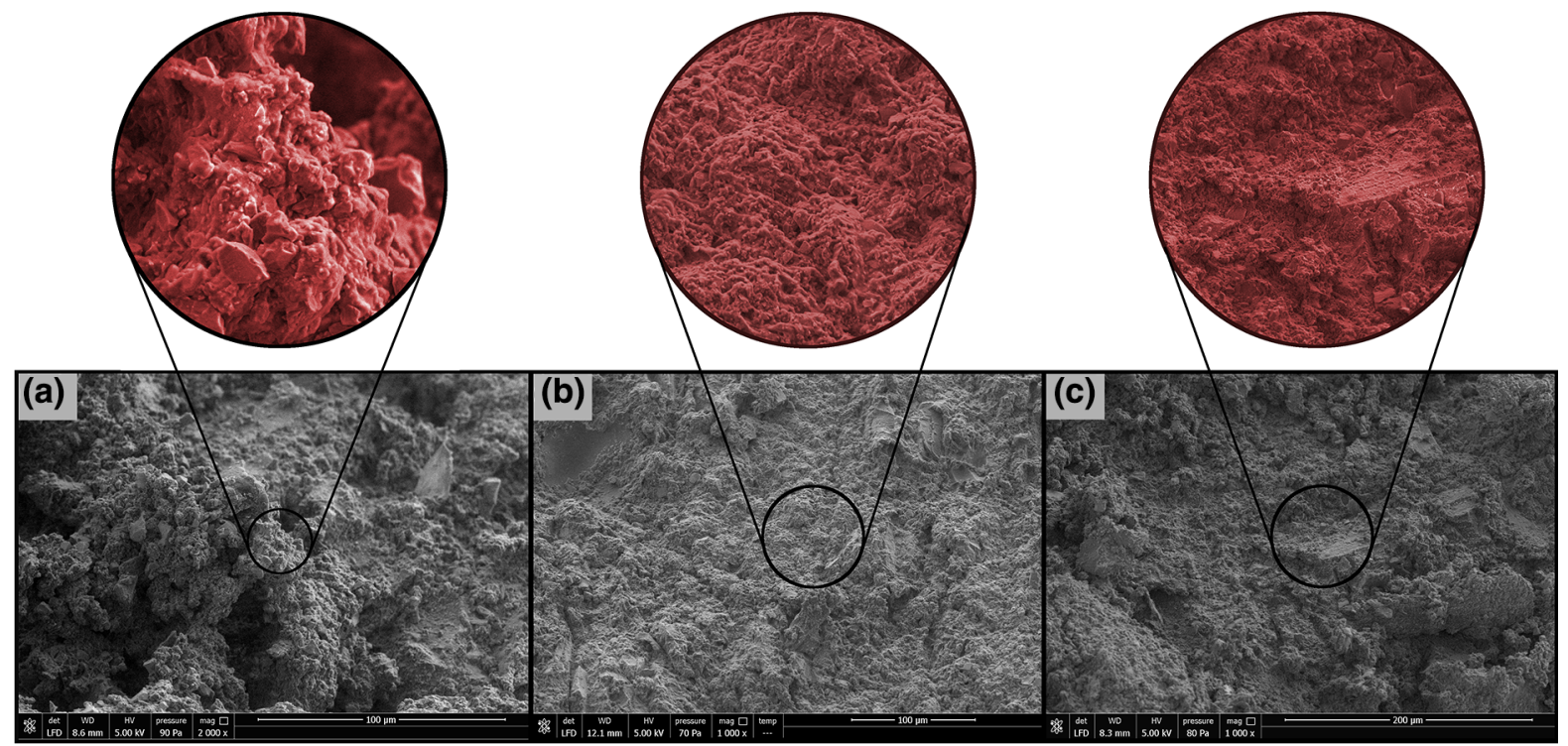

Fig. 13 SEM images of the monolith showing its regenerative capability a prior to compression, $\mathbf{b}$ after being subjected to a quasistatic compressive stress of $10 \mathrm{MPa}$, and $\mathbf{c}$ after regeneration

\section{Conclusions}

In this work, we examined the durability of the newly developed superhydrophobic surfaces proposed in Part I to potentially combat the transmission and spread of COVID-19. Thermal degradation, chemical attack and mechanical abrasion tests were conducted, and the superhydrophobic surface characteristics were re-tested and re-evaluated. Our extensive test results revealed that the developed epoxy-based and siliconebased nanocomposites can maintain their superhydrophobicity after being subjected for $3 \mathrm{~h}$ to elevated temperatures of $150{ }^{\circ} \mathrm{C}$ and $350{ }^{\circ} \mathrm{C}$, respectively. The developed surfaces also preserved their characteristics after being immersed in alkaline and acidic solutions ( $\mathrm{pH}$ values 1-13) for $3 \mathrm{~h}$. In order to assess the mechanical durability of epoxy-based nanocomposites, samples were subjected to abrasion tests, and they were capable of maintaining their superhydrophobicity for 90 abrasion cycles. Moreover, the regeneration capabilities of the silicone-based nanocomposites were demonstrated by subjecting the monoliths to destructive compressive loads (up to $10 \mathrm{MPa}$ ), and then the high CA and low SA were easily restored through abrasion. These results verify the ability of these surfaces to survive harsh service conditions and their potential use to combat the transmission and spread of infected surfaces. It is important to note that we have neither examined the combined effect of these parameters upon the surface characteristics of the developed superhydrophobic surfaces nor the prolonged exposure to these conditions (aging), which are considered beyond the scope of the current study.

Acknowledgements This study was financially supported by the Natural Sciences and Engineering Research Council of Canada (Project No. RGPIN-2018-03804).

\section{References}

BaratiDarband, G., Aliofkhazraei, M., Khorsand, S., Sokhanvar, S., Kaboli, A.: Science and engineering of superhydrophobic surfaces: review of corrosion resistancechemical and mechanical stability. Arab. J. Chem. 13, 1763-1802 (2020). https://doi.org/10.1016/j.arabjc.2018. 01.013

Cha, S.C., Her, E.K., Ko, T.J., Kim, S.J., Roh, H., Lee, K.R., Oh, K.H., Moon, M.W.: Thermal stability of superhydrophobic, nanostructured surfaces. J. Colloid Interface Sci. 391, 152-157 (2013). https://doi.org/10.1016/j.jcis.2012.09.052

Cui, X., Lin, X., Liu, C., Yang, R., Zheng, X., Gong, M.: Fabrication and corrosion resistance of a hydrophobic micro-arc oxidation coating on AZ31 Mg alloy. Corros. Sci. 90, 402-412 (2015). https://doi.org/10.1016/j.corsci. 2014.10.041

Ellinas, K., Tserepi, A., Gogolides, E.: Durable superhydrophobic and superamphiphobic polymeric surfaces and their applications: a review. Adv. Colloid Interface Sci. 250, 132-157 (2017). https://doi.org/10.1016/j.cis.2017. 09.003 
Elzaabalawy, A., Verberne, P., Meguid, S.A.: Multifunctional silica-silicone nanocomposite with regenerative superhydrophobic capabilities. ACS Appl. Mater. Interfaces. 11, 42827-42837 (2019). https://doi.org/10.1021/acsami. $9 \mathrm{~b} 15445$

Falde, E.J., Yohe, S.T., Colson, Y.L., Grinstaff, M.W.: Superhydrophobic materials for biomedical applications. Biomaterials 104, 87-103 (2016). https://doi.org/10.1016/j. biomaterials.2016.06.050

Gralinski, L.E., Menachery, V.D.: Return of the coronavirus: 2019-nCoV. Viruses 12, 1-8 (2020). https://doi.org/10. 3390/v12020135

Ishizaki, T., Hieda, J., Saito, N., Saito, N., Takai, O.: Corrosion resistance and chemical stability of super-hydrophobic film deposited on magnesium alloy AZ31 by microwave plasma-enhanced chemical vapor deposition. Electrochim. Acta 55, 7094-7101 (2010). https://doi.org/10.1016/j. electacta.2010.06.064

Jaggessar, A., Shahali, H., Mathew, A., Yarlagadda, P.K.D.V.: Bio-mimicking nano and micro-structured surface fabrication for antibacterial properties in medical implants. J. Nanobiotechnol. 15, 1-20 (2017). https://doi.org/10. 1186/s12951-017-0306-1

Jeevahan, J., Chandrasekaran, M., BrittoJoseph, G., Durairaj, R.B.: Mageshwaran G (2018) Superhydrophobic surfaces: a review on fundamentals, applications, and challenges. J. Coat. Technol. Res. 15, 231-250 (2018). https://doi.org/ 10.1007/s11998-017-0011-x

Katoh, I., Tanabe, F., Kasai, H., Moriishi, K., Shimasaki, N., Shinohara, K., Uchida, Y., Koshiba, T., Arakawa, S., Morimoto, M.: Potential risk of virus carryover by fabrics of personal protective gowns. Front. Public Heal. 7, 3-8 (2019). https://doi.org/10.3389/fpubh.2019.00121

Milionis, A., Loth, E., Bayer, I.S.: Recent advances in the mechanical durability of superhydrophobic materials. Adv. Colloid Interface Sci. 229, 57-79 (2016). https://doi.org/ 10.1016/j.cis.2015.12.007

Mortazavi, V., Khonsari, M.M.: On the degradation of superhydrophobic surfaces: a review. Wear 372-373, 145-157 (2017). https://doi.org/10.1016/j.wear.2016.11.009
Saleema, N., Farzaneh, M.: Thermal effect on superhydrophobic performance of stearic acid modified $\mathrm{ZnO}$ nanotowers. Appl. Surf. Sci. 254, 2690-2695 (2008). https://doi.org/10. 1016/j.apsusc.2007.10.004

Shin, S., Seo, J., Han, H., Kang, S., Kim, H., Lee, T.: Bioinspired extreme wetting surfaces for biomedical applications. Materials (Basel) (2016). https://doi.org/10.3390/ ma9020116

Tomšič, B., Simončič, B., Orel, B., Černe, L., Tavčer, P.F., Zorko, M., Jerman, I., Vilčnik, A., Kovač, J.: Sol-gel coating of cellulose fibres with antimicrobial and repellent properties. J. Sol-Gel. Sci. Technol. 47, 44-57 (2008). https://doi.org/10.1007/s10971-008-1732-1

Van Doremalen, N., Bushmaker, T., Morris, D., Holbrook, M., Gamble, A., Williamson, B., Tamin, A., Harcourt, J., Thornburg, N., Gerber, S., Lloyd-Smith, J.: Aerosol and surface stability of SARS-CoV-2 as compared with SARSCoV-1. N. Engl. J. Med. 382(16), 1564-1567 (2020)

Yang, P., Wang, X.: COVID-19: a new challenge for human beings. Cell. Mol. Immunol. (2020). https://doi.org/10. 1038/s41423-020-0407-x

Yeerken, T., Wang, G., Li, H., Liu, H., Yu, W.: Chemical stable, superhydrophobic and self-cleaning fabrics prepared by two-step coating of a polytetrafluoroethylene membrane and silica nanoparticles. Text. Res. J. 89, 4827-4841 (2019). https://doi.org/10.1177/0040517519842795

Zhang, X., Wang, L., Levänen, E.: Superhydrophobic surfaces for the reduction of bacterial adhesion. RSC Adv. 3, 12003-12020 (2013). https://doi.org/10.1039/c3ra40497h

Zhao, L., Hong, C., Lin, L., Wu, H., Su, Y., Zhang, X., Liu, A.: Controllable nanoscale engineering of vertically aligned MoS2 ultrathin nanosheets by nitrogen doping of 3D graphene hydrogel for improved electrocatalytic hydrogen evolution. Carbon N. Y. 116, 223-231 (2017). https://doi. org/10.1016/j.carbon.2017.02.010

Publisher's Note Springer Nature remains neutral with regard to jurisdictional claims in published maps and institutional affiliations. 